\title{
Atomic force microscopy techniques for nanomechanical characterization: a polymeric case study \\ --Manuscript Draft--
}

\begin{tabular}{|c|c|}
\hline \multicolumn{2}{|l|}{ Manuscript Number: } \\
\hline Full Title: & $\begin{array}{l}\text { Atomic force microscopy techniques for nanomechanical characterization: a polymeric } \\
\text { case study }\end{array}$ \\
\hline Article Type: & Original Paper \\
\hline Corresponding Author: & $\begin{array}{l}\text { Marco Rossi } \\
\text { ITALY }\end{array}$ \\
\hline \multicolumn{2}{|c|}{$\begin{array}{l}\text { Corresponding Author Secondary } \\
\text { Information: }\end{array}$} \\
\hline \multicolumn{2}{|c|}{ Corresponding Author's Institution: } \\
\hline \multicolumn{2}{|c|}{$\begin{array}{l}\text { Corresponding Author's Secondary } \\
\text { Institution: }\end{array}$} \\
\hline First Author: & Melania Reggente \\
\hline \multicolumn{2}{|c|}{ First Author Secondary Information: } \\
\hline \multirow[t]{8}{*}{ Order of Authors: } & Melania Reggente \\
\hline & Marco Rossi \\
\hline & Livia Angeloni \\
\hline & Emanuela Tamburri \\
\hline & Massimiliano Lucci \\
\hline & Ivan Davoli \\
\hline & Maria Letizia Terranova \\
\hline & Daniele Passeri \\
\hline \multicolumn{2}{|c|}{ Order of Authors Secondary Information: } \\
\hline Abstract: & $\begin{array}{l}\text { Atomic Force Microscopy (AFM) is a versatile tool to perform mechanical } \\
\text { characterization of surface samples at the nanoscale. In this work, we review two of } \\
\text { such methods, namely contact resonance AFM (CR-AFM) and } \\
\text { torsional harmonics AFM (TH-AFM). First, such techniques are illustated and their } \\
\text { applicability on materials with elastic moduli in different ranges are discussed, together } \\
\text { with their main advantages and limitations. Then, a case study is presented where we } \\
\text { report the mechanical characterization using both CR-AFM and TH-AFM of polyaniline } \\
\text { and polyaniniline doped with nanodiamond particles tablets prepared by a pressing } \\
\text { process. We determined the indentation modulus values of their surfaces which were } \\
\text { found in fairly good agreement, thus demonstrating the accuracy of the techniques. } \\
\text { Finally, } \\
\text { the determined surface elastic moduli have been compared to the bulk ones measured } \\
\text { through standard indentation testing. }\end{array}$ \\
\hline
\end{tabular}




\title{
Atomic force microscopy techniques for nanomechanical characterization: a polymeric case study
}

Melania Reggente - Marco Rossi - Livia Angeloni - Emanuela Tamburri - Massimiliano Lucci - Ivan Davoli Maria Letizia Terranova - Daniele Passeri

\begin{abstract}
Atomic Force Microscopy (AFM) is a versatile tool to perform mechanical characterization of surface samples at the nanoscale. In this work, we review two of such methods, namely contact resonance AFM (CR-AFM) and torsional harmonics AFM (TH-AFM). First, such techniques are illustrated and their applicability on materials with elastic moduli in different ranges are discussed, together with their main advantages and limitations. Then, a case study is presented where we report the mechanical characterization using both CR-AFM and TH-AFM of polyaniline and polyaniniline doped with nanodiamond particles tablets prepared by a pressing process. We determined the indentation modulus values of their surfaces which were found in fairly good agreement, thus demonstrating the accuracy of the techniques. Finally, the determined surface elastic moduli have been compared to the bulk ones measured through standard indentation testing.
\end{abstract}

Keywords Contact resonance AFM - Atomic force acoustic microscopy - Torsional harmonics AFM - HarmoniX polyaniline - nanodiamond - elastic modulus

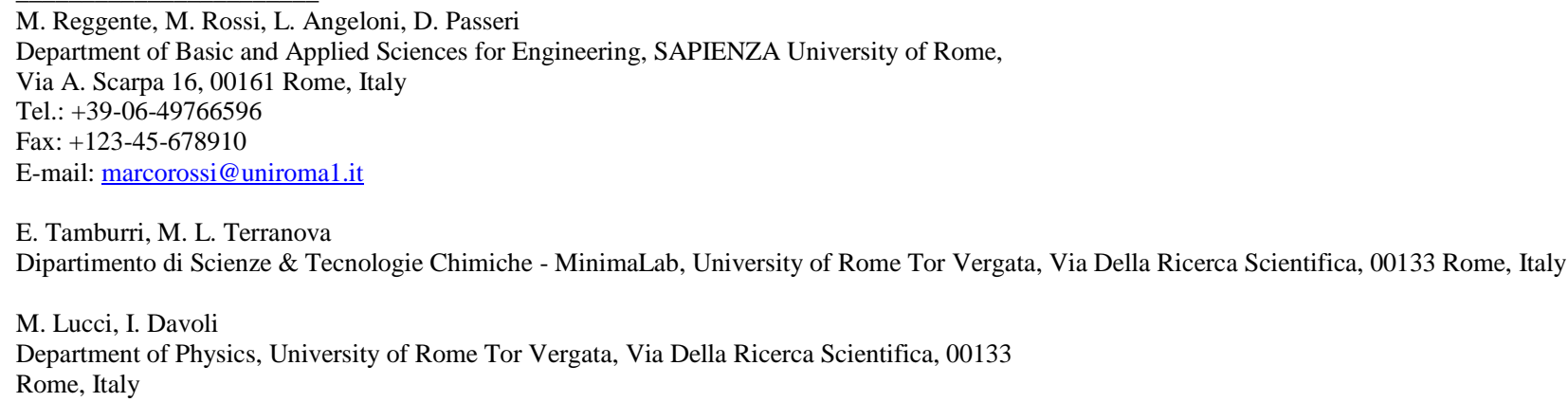




\section{Introduction}

In the field of nanotechnology the development of innovative and nondestructive characterization techniques plays a crucial role. Indeed, the characterization of nanostructured hybrid materials (e.g., thin films and nanocomposites) and devices, requires the capability of acquiring maps of the local mechanical properties at nanoscale. Nanoindentation is the most common method for determining the mechanical properties of thin films. However, its applicability is strictly limited by the thickness of the sample. Furthermore, its poor spatial resolution does not allow the reconstruction of an accurate distribution of the sample surface mechanical properties. For this reason, alternative methods, based on atomic force microscopy (AFM), have been developed. By exploiting the high resolution of the AFM, maps of the surface mechanical properties (i.e., indentation modulus) can be achieved. Among these techniques, AFM nanoindentation [1] is the simplest method used to evaluate the local mechanical properties of both stiff and soft samples [2-5]. AFM nanoindentation consists in locally recording force-indentation (FI) curves. If the FI curves are collected at each point of the scanning area, a stiffness map of the sample is obtained in the so called force volume mode (FVM) [6]. Due to the low of resolution and the long acquisition time required by this technique, different AFM based methods have been established. In particular, contact resonance AFM (CR-AFM) and torsional harmonics AFM (TH-AFM) can be used to perform both qualitative and quantitative maps of the mechanical properties of the sample surfaces with nanometric lateral spatial resolution. CR-AFM is a well established technique for the characterization of hard elastic materials [79]. Furthermore, recently it has been employed to investigate the indentation moduli and the viscoelastic properties (i.e., storage and loss moduli) [10-13] of soft polymeric films. Instead, TH-AFM is one of the latest AFM mode specifically developed to characterize soft matters. It operates in tapping mode using a T-shaped cantilevers with an out-of-axis tip which periodically indents the surface during the scanning. By collecting the FI curves produced by the periodical indentation of the tip into the sample, maps of the mechanical properties of the surface sample are achieved [14-17]. In previous studies, by adopting the AFM techniques above mentioned (i.e., AFM nanoindentation, CR-AFM, THAFM) we deeply analyzed the mechanical properties of hybrid nanocomposite materials based on conductive polymers, i.e., polyaniline (PANI) and poly(3,4-ethylenedioxythiophene) (PEDOT) filled with nanodiamond (ND) particles [5, 17-22]. In particular, we have pointed out that ND is able to induce a high structural order of the polymer chains which organize themselves in the form of nanofibrils, nanoglobules or nanoparticles depending on the experimental conditions of the synthesis. On such materials, we performed accurate material characterizations at the nanoscale $[5,17,18,21$, 22].

Considering the results obtained so far, in the present study we investigated how the mechanical properties of the single nanostructures can rule the surface mechanical properties of a macroscale sample, especially when it consists of a dense and forced packing of the individual nanostructures. For this purpose, we firstly synthesized polyaniline (PANI) and polyaniline doped with nanodiamond (ND) particles (PANI-ND) by a precipitation method and then we have prepared PANI and PANI-ND tablets by a pressing process conducted at 200 bar. Thus, we have investigated the effect of the compression of the polymer chains on both the bulk and surface mechanical properties of the two final tablets, by using standard nanoindentation for the bulk properties and both TH-AFM and CR-AFM for the surface ones.

\section{Techniques}

\subsection{Torsional harmonics atomic force microscopy}

In TH-AFM, the sample surface is imaged in tapping mode. The cantilever is oscillating at (a frequency close to) its first free flexural resonance $f_{01}$. In case of compliant samples, during a portion of each oscillation cycle the tip indents the sample surface. As a consequence, during each oscillation cycle the cantilever deflection signal contains a complete loading/unloading force versus penetration curve which is repeated with period $T=1 / f_{01}$. Therefore, the periodic force versus penetration curve could be reconstructed through inverse Fourier transform from the harmonic components at frequencies $N \times f_{01}$ of the deflection signal. Nevertheless, such a procedure is limited by to the poor signal-to-noise ratio due to the presence of the higher flexural modes of the cantilever [23,24]. To overcome this practical limitation, a T-shaped cantilever with out-of-axis tip is used. The tip-sample contact produces a torque which excites the torsion of the cantilever, enhanced by its geometry. Analogously to the deflection signal, the cantilever torsional signal contains a complete loading/unloading force versus penetration curve, periodic with period $T=1 / f_{01}$, which can be reconstructed through inverse Fourier transform analysis. In this case, the use of the torsional signal ensures a higher signal-to-noise ratio since the first torsional resonance frequency $t_{01}$ is about 20 times higher than $f_{01}$ and thus at least 20 harmonic components of the signal are located in an undistorted spectral region [23, 24]. As a result, during the tapping, forcedistance curves are obtained at each point of the scanned area. After proper calibrations, such curves are analyzed in order to map the sample indentation modulus $M_{s}$ through the fitting of the unloading portion of the curve with the Derjaguin-Muller-Toporov (DMT) model [25]. Additional parameters mapped during the scanning are the peak force, the tip-sample adhesion force, and the dissipated energy during each indentation cycle [17]. In particular, the quantitative mapping of $M_{s}$ requires the knowledge of $R_{t}$. Alternatively, a sample with known elastic modulus can be 
used as a reference, which is the approach used in this work.

Being based on AFM tapping mode and thus not requiring the continuous contact between tip and sample, TH-AFM finds natural application in the nanomechanical study of soft samples, like polymers or biological structures. The materials which have been effectively investigated by TH-AFM have indentation modulus ranging approximately from $10 \mathrm{MPa}$ up to $10 \mathrm{GPa}$ [24], including highly oriented pyrolytic graphite (HOPG) [24], polymers [15,24], polymer blends [17, 24], polymer-based (nano)composites [14, 21], pigment coatings [26], amyloid fibrils [27], bacterial nanowires [28]. Although the range of investigable elastic moduli is no as wide as those of other AFM-based techniques, TH-AFM is particularly suitable to acquire simultaneous high resolution (i.e., $256 \times 256$ or $512 \times 512$ points) and high-speed (e.g., a few minutes) mechanical images of soft samples, which conversely may represent challenging materials for other AFM-based methods, mainly for those working in contact mode. Also, TH-AFM probes the sample down to a few tens of nanometers from the surface. As a consequence, mechanical properties of the bulk may be not accessible and the comparison between TH-AFM data and those from techniques which probe the sample down to bigger penetration depths (such as depth sensing indentation) may be misleading. On the other hand, the shallow penetration depth makes TH-AFM particularly suitable for the study of mechanical properties of polymeric ultrathin films.

\subsection{Contact resonance atomic force microscopy}

CR-AFM technique has been extensively described in previous works [29, 30]. Briefly, the AFM experimental apparatus is equipped with a piezoelectric transducer that is coupled to the back of the sample. The piezoelectric transducer excites out-of-plane oscillation at ultrasonic frequencies of the sample surface. Being the AFM tip in contact with the sample surface, such oscillation results in the high frequency modulation of both the cantilever deflection and the tip-sample penetration depth. By analyzing the high frequency components of the cantilever deflection signal through the AFM electronics, one can measure the resonance frequencies of the sample-tip-cantilever system - namely, the contact resonance frequencies (CRFs) $-f_{n}{ }^{\prime} s$, where the subscript $n$ indicates the $n$-th mode. The experimentally determined values of $f_{n}$ 's can be used to evaluate the elastic properties of the surface of the sample. In particular, the indentation modulus of the samples $\left(M_{s}\right)$ can be obtained, which is defined as $E_{s}=\left(1-2 v_{s}^{2}\right)$ for isotropic materials, where $E_{s}$ and $v_{s}$ are the Young's modulus and the Poisson ratio of the sample, respectively. To this aim, the experimentally measured values of $f_{n}$ 's are analyzed assuming a suitable model for the cantilever, the most commonly used of which is depicted in Fig. 1a. The cantilever is approximated as a uniform beam with length $L$ and rectangular cross section with thickness $t$ and width $w$, with its main axis parallel to the sample surface. The tip is placed at distance $L_{1}$ from the cantilever clamped end, so that the ratio $r=L_{1} / L$ can be defined. The tip-sample contact is modeled as a linear spring, the elastic constant of which is the contact stiffness $k^{*}$, which accounts for the mechanical properties of the sample surface, i.e., $M_{s}$. The damping and the effect of tip-sample lateral forces are neglected. The characteristic equation of the system in Fig. 1a, whose explicit analytical expression is easily obtained and can be found elsewhere [29], shows that the values of $f_{n}^{\prime} s$ are fixed by $r, k^{*}$ and a geometrical parameter $c_{c}$ characteristic of each cantilever. In particular, it is observed that for fixed values of $r$ and $c_{c}$, the higher $k^{*}$ the higher $f_{n}{ }^{\prime} s$. Such a characteristic equation can be inverted so that $k^{*}=k^{*}\left(f_{n}, r\right)$ is obtained as a function of both the generic $f_{n}$ and $r$. To evaluate $r$ that is generally unknown, the CRFs corresponding to two different modes are measured (say $f_{n}$ and $f_{m}$, with $n \neq m$ ). $r$ is obtained as the value in correspondence of which the two CRFs give the same contact stiffness, i.e., by solving $k^{*}\left(f_{n}, r\right)=k^{*}\left(f_{m}, r\right)$. Thus $k^{*}$ is determined, and can be used to evaluate $M_{t}$, assuming that the values of the indentation modulus of the tip $M_{t}$, the tip radius $R_{t}$, and the normal load applied by the cantilever $F_{N}$ are known. Indeed, in case of parabolical tip, $M_{s}$ is evaluated from $k^{*}$ using the relation

$$
\frac{1}{k^{* 3 / 2}}=\frac{1}{\sqrt{6 R_{t} F_{N}}}\left(\frac{1}{M_{S}}+\frac{1}{M_{t}}\right)
$$

$F_{N}$ can be easily calculated from the static cantilever deflection. $R_{t}$ is generally evaluated by calibration of the technique using a reference sample with known mechanical properties. $M_{t}$ is either assumed as the theoretical indentation modulus of the material forming the tip [30, 31] or independently measured using a second reference material [32]. The latter approach has been demonstrated to overcome also the limitation due to neglecting the tipsample lateral forces, which represents a major source of inaccuracy in CR-AFM measurements especially when characterizing polymers [33]. Indeed, a more comprehensive model of the system is depicted in Fig. 1b, where the cantilever is assumed tilted with respect to the surface of an angle $\alpha$ and the tip height $h_{t}$ is considered [34-36]. Moreover, the lateral contact stiffness $k_{\text {lat }}^{*}$ is introduced, which accounts for the tip-sample lateral coupling, i.e., the sample shear modulus $G_{s}=\frac{1}{2} E_{s} /\left(1+v_{s}\right)$ [36]. For practical purposes, it has been demonstrated that $k_{\text {lat }}^{*}$ can be neglected and experimental data can be analyzed through the model in Fig. 1a provided that fictitious values of $M_{t}$ and $r$ are assumed [33]. Conversely, including lateral coupling in the model allows one to analyze experimental data assuming the real values of such parameters. In this case, the values of all the parameters in the model are fixed and $k^{*}\left(f_{n}, r\right)=k^{*}\left(f_{m}, r\right)$ is not imposed. As a consequence, different values of $k^{*}$ may be obtained in correspondence of different modes $[11,13]$. This may represent an artifact originated from approximation in the model as well as a real 
effect reflecting frequency-dependent mechanical parameters of the sample. Based on AFM contact mode, CR-AFM is particularly suitable for the nanomechanical characterization of relatively stiff materials, including diamondlike carbon (DLC) coatings [7, 9], ultrathin films [10], piezoelectric materials [37], single crystals [30], nanobelts and nanotubes [38, 39], epoxy-based nanocomposites [40-42]. Recently, CR-AFM has been extended to softer samples, i.e., polymers. In particular, CR-AFM allows one to determine the storage and loss moduli of viscoelastic materials from the analysis of the contact resonance frequencies and the corresponding quality factors $[11,13]$. Finally, such parameters have been mapped on the surface of polymeric blends [12,43]. Notwithstanding these results, the application of CR-AFM to soft materials still remains challenging due to the fact that it involves the continuous tip sample contact during all the image acquisition which is actually quite time consuming even for not too high resolution images (e.g., 10-20 minutes for $128 \times 128$ points images). Moreover, some difficulties in the quantitative analysis of CR-AFM data may arise from the need for more accurate models where lateral forces or dissipation are not neglected.

\section{A case study: polyaniline and polyaniline-nanodiamond composites}

In this section we report the results of the functional characterization of polyaniline (PANI) and polyaniline doped with nanodiamond (ND) particles (PANI-ND). The aim of the study is to determine how the introduction of detonation NDs modulates the elastic surface properties of the samples when they are prepared by a pressing process. To this end, the indentation moduli of PANI and PANI-ND samples have been calculated by employing two different AFM based techniques, i.e., TH-AFM and CR-AFM previously described.

\subsection{Materials}

PANI and PANI-ND materials were synthesized following a protocol optimized in previous studies. Namely, a precipitation polymerization of aniline monomer was carried out in acidic aqueous solution using an anionic surfactant, i.e., sodium dodecyl sulfate (SDS), as dopant and dispersing agent of ND particles in the case of the PANI-ND composite. The complete characterization of the obtained PANI and PANI-ND samples was performed using optical microscopy, both transmission and scanning electron microscopy (TEM and SEM, respectively), Raman spectroscopy, reflection high energy electron diffraction (RHEED), X-ray diffraction (XRD), and thermo gravimetric analysis (TGA), which can be found elsewhere [18]. Tablet samples $1 \mathrm{~mm}$ thick were prepared by pressing, at $200 \mathrm{bar}, 100 \mathrm{mg}$ of PANI and PANI-ND.

\subsection{Equipment}

CR-AFM experiments have been performed using an AFM setup (Solver, NTMDT, Russia) equipped with a silicon cantilever (CSG10, NT-MDT, Russia). The cantilever used in the experiments has a length of $250 \mu \mathrm{m}$, width of $35 \mu \mathrm{m}$, thickness of $1 \mu \mathrm{m}$, and nominal spring constant $k_{c}=0.1 \mathrm{~N} / \mathrm{m}$. The cantilever has been used after extensive scanning of the surface of hard samples in order to severely wear the tip, as large tip radius and thus large tip-sample contact radius values reduce the stress under the tip and consequently diminish the damaging of soft samples during AFM contact mode imaging. For TH-AFM measurements, a standard AFM apparatus (Dimension Icon, Bruker Inc.) has been employed which was equipped with T-shaped cantilevers (HMX10, Bruker Inc.) with nominal spring constant $k_{c}=4$ $\mathrm{N} / \mathrm{m}$. Brand new cantilevers have been used after some preliminary scans of the polymeric samples in order to allow the tip radius to stabilize. Both CR-AFM and TH-AFM experiments have been performed in air and at room temperature. In both the techniques, quantitative measurements have been performed using a blend of polystyrene (PS) and low density polyethylene (LDPE) reference sample (PS-LDPE, Bruker Inc.) with known mechanical properties for calibration purposes. The PS-LDPE sample consists of a matrix of PS in which there are dispersed round-shaped regions of LDPE, deposited onto a silicon substrate. The PS matrix has an elastic modulus equal to 2 GPa, while the elastic modulus of LDPE regions is about $100 \mathrm{MPa}$.

The indentation modulus and hardness measurements have been performed by Nano Test Micro Materials Ltd, using a diamond Berkovitch tip. For each sample, an array of $10 \times 10$ points have been probed, covering a surface of a few microns squared, by applying a maximum load of $5 \mathrm{mN}$, holding time of $10 \mathrm{~s}$, indentation depth doing range between 500 and $1200 \mathrm{~nm}$, penetration speed/load control of $0.2 \mathrm{mN} / \mathrm{s}$. The instrumental uncertainty of the indenter is approximately $2 \%[44,45]$.

\subsection{Results and discussion}

Preliminarily to the mechanical characterization of the samples, the morphology of the samples has been characterized in standard AFM tapping mode. In Fig. 2a and $2 \mathrm{~b}$ two typical topographical images of PANI are reported at two different magnifications after subtraction of polynomial background in order to emphasize the different nanoscale features, which show a granular structure. Conversely, on the surface of PANI-ND fibrillar features are visible (Fig. 2c and $2 \mathrm{~d}$ ) which are triggered by the presence of the ND particles, as already observed by Tamburri et al. [18].

In order to validate the results retrieved with TH-AFM, by verifying the stability and the accuracy of the technique, 
several preliminary measurement sessions have been carried out under different operating conditions (i.e., different cantilevers of the same type and set-point values). In each experiment, the characterization of the PS-LDPE reference sample has been performed twice, before and after the measurement on the investigated samples. In each experimental session, all the samples have been scanned under the same operating conditions (i.e., same set-point, drive-frequency and drive-amplitude values). The acquired indentation modulus maps, initially expressed in arbitrary units, have been calibrated on the PS-LDPE reference sample according to the procedure supplied by the producer. In particular, the indentation modulus of the PS matrix has been used for the calibration of the technique while the LDPE has been used as a control material. For each sample, the statistics were calculated on the collected indentation modulus maps to assign the values of indentation moduli and corresponding uncertainties. As an example of the typical results of a THAFM measurement session, the histograms of the indentation moduli of the investigated and reference samples are reported in Fig. 3. It should be noted that the obtained value of the indentation modulus of the LDPE is in fairly good agreement with the expected one even though slightly lower, indicating a not perfect calibration for low modulus values. Nevertheless, since the moduli of the investigated PANI and PANI-ND samples are of the same order of magnitude of those of the PS, the calibration can be considered accurate enough in the range of interest. The obtained indentation modulus values of PANI and PANI-ND are reported in Table 1.

CR-AFM is a two steps technique. In the first step the free resonance frequencies of the cantilever oscillating in air $\left(f_{0 n}\right)$ are measured in order to experimentally determine the characteristic parameters of the cantilever (i.e., the $c_{c}$ parameter). In Tab. 2 the first four measured natural frequencies are reported and compared to those calculated supposing the cantilever as a uniform rectangular beam [29]. It should be noted that the ratio between the calculated and the measured values of the frequencies keeps constant for each mode of the cantilever. Indeed, the used cantilever is rectangular and the differences between measured and calculated free resonance frequencies result from the deviation of the actual geometrical parameters from the nominal ones. The ratio between calculated and experimental free resonance frequencies can be used to individuate the correct free flexural resonances in case of the presence of multi-peaks. In this work, CR-AFM nanomechanical measurements have been performed using only the first and the third resonance frequencies. The parameter $c_{c}$ was determined from the values of the first $\left(f_{01}\right)$ and third $\left(f_{03}\right)$ free resonance frequencies through the relation

$$
c_{c}=\frac{k_{n} L}{\sqrt{f_{0 n}}}
$$

where $k_{n} L$ are eigenvalues of each mode which are well known from literature [29]. In particular, the value of $c_{c}$ was determined for each mode, so that $c_{c 1}$ and $c_{c 3}$ were calculated from $f_{01}$ and $f_{03}$, respectively.

In the second step of the procedure the tip is brought in contact with the sample surface which is set into oscillation through a piezoelectric transducer coupled with the sample back side. Maps of the first and third CRFs $\left(f_{1}\right.$, $f_{3}$ ) of the investigated samples are recorded and semi-quantitative maps of the samples stiffness was achieved. In order to extract quantitative information about the indentation modulus of the investigated samples and eliminate the uncertainty related to the mechanical $\left(M_{t}\right)$ and geometrical $\left(R_{t}\right)$ properties of the tip, calibration has been performed on the PS-LDPE reference sample. Furthermore, to guarantee the stability of the experimental session, it has been tested twice, before and after the two investigated samples. As en examples, in Fig. 4 a typical CR-AFM map of $f_{1}$ on PSLDPE is reported, together with a profile of the first CRF extracted from the map in correspondence of the line. As expected, in correspondence of the LDPE regions the values of the frequencies decrease, as a result of the lower value of indentation modulus and thus of $k^{*}$. To reduce the possibility of tip damaging between the two investigated samples, we purposely investigated the samples following the decreasing order of expected stiffness, i.e., starting from the sample which we expected to be the stiffer one. In this way, we were able to monitor the performances of our technique during the whole experimental session. Furthermore, to prevent tip wear, we avoided long scans of the surfaces limiting the acquisition to a number of lines which ensured enough points to make a significant statistics of the results. For all the reference and investigated samples, $f_{1}$ and $f_{3}$ have been measured by maintaining constant the cantilever deflection set point, i.e., thus the applied load. Statistics of the CRFs maps have been used to determine mean values and corresponding uncertainties of the indentation modulus of the samples. As expected, CRFs measured on the PANI-ND samples are higher than that measured on the undoped PANI, indicating that the former is stiffer than the latter.

In the post-experiment data analysis, CRFs have been analyzed with a suitable method in order to calculate the contact stiffness of each sample $\left(k_{s}^{*}\right)$ and to quantitative determine the value of their indentation modulus $\left(M_{s}\right)$. To this aim, the simplest model depicted in Fig. 1a is the most widespread one to analyze CR-AFM data. Nevertheless, it has been recently shown that on polymeric materials the presence of tangential spring constant $k_{\text {lat }}^{*}$, which is related to the reduced sample shear modulus [36], may dramatically affect the calibration procedure [33]. Thus, we analyzed our CRAFM experimental data by modeling the contact between the tip and the sample by considering both a vertical and a tangential spring (with spring constant defined by $k^{*}$ and $k_{\text {lat }}^{*}$, respectively), i.e., assuming the model depicted in Fig. 1b. In particular, considering realistic or experimentally determined values for the parameters of the model in Fig. 1b, we simulated the $f_{1}$ and $f_{3}$ values in a wide range of $k^{*} / k_{c}$ values, in order to determine the values of $k_{s}^{*}$ for each sample from the measured values of CRFs. In details, since the $M_{t} \gg M_{s}$, the ratio $k_{\text {lat }}^{*} / k^{*}$, was determined by the following expression [34]:

$$
\frac{k_{l a t}^{*}}{k^{*}} \approx \frac{2\left(1-v_{S}\right)}{2-v_{S}}
$$


The value of the Poisson ratio, $v_{s}$, has been approximated to 0.4 for all the measured samples. The dimensions of the cantilever have been assumed as the nominal ones supplied by the manufacturer and the beam was inclined by an angle $\alpha=11 \mathrm{deg}$ with respect to the sample surface. The tip, with nominal height $h=15 \mu \mathrm{m}$, was placed at a distance $L_{1}$ from the cantilever clamped end and its position was defined by the $r=L_{1} / L=0.985$, experimentally evaluated by scanning electron microscopy (SEM) analysis. The first and third CRFs have been determined by numerically solving the characteristic equation of the system (reported in [36]) for values of $k^{*} / k_{c}$ ranging between 1 and $10^{4}$. In this way, curves of the $f_{1}$ and $f_{3}$ CRFs versus the normalized contact stiffness $k^{*} / k_{c}$ are obtained which are reported in Fig. 5 (solid line). For comparison, for the same values of $k^{*} / k_{c}$ the CRFs determined using the simplest model which neglects $k_{\text {lat }}^{*}$, are reported (dotted lines). It should be explicitly observed that the simple model is unable to predict the experimental CRFs, as the well known saturation of the CRFs for large stiffness $k^{*} / k_{c}$ [29] corresponds to frequency values lower than those measured on the stiffer samples. Conversely, the CRFs experimentally determined on all the sample are predicted using the model (Fig. 1b) which takes into account lateral tip sample coupling. From these curves, two values of the $k^{*}$, one for each CRFs recorded, have been extracted for each samples. From the values of $k^{*} / k_{c}$ those of $M_{s}$, have been calculated. As the inclusion of $k_{\text {lat }}^{*}$, in the model allows one to consider the real values of the parameters [33], being $M_{t} \gg M_{s}$ Eq. (1) can be approximated as

$$
\left(k^{*}\right)^{3 / 2}=\sqrt{6 R_{t} F_{N}} M_{s}=A M_{s},
$$

indicating a direct proportionality between $k^{* 3 / 2}$ and $M_{S}$ (for both the two chosen vibration modes). The proportionality coefficient $A$ has been determined on the PS sample, independently for each of the analyzed modes, and the moduli of the LDPE control materials and of PANI and PANI-ND are finally retrieved. We want to explicitly report that the indentation modulus of the LDPE control material retrieved using $f_{1}\left(M_{L D P E}=130 \mathrm{MPa}\right)$ fairly matches the reference value, while the one retrieved using $f_{3}\left(M_{L D P E}=660 \mathrm{MPa}\right)$ is significantly higher. This result can be rationalized considering that the LDPE islands are some tens of nanometers thick and are surrounded by PS. In TH-AFM, the indentation depth and the contact radius are a few nanometers and, therefore, the technique is sensitive to the mechanical properties in a volume under the surface with a depth usually not exceeding a few tens of nanometers. Conversely, as CR-AFM is performed using worn tips with large radius, larger tip-sample contact radii are involved. In the case of PS, the contact radius can be evaluated as large as some tens of nanometers. In addition, in CR-AFM higher modes are more sensitive to buried features due to the increased effective stiffness [46]. Therefore, the third CRF is more sensitive to the presence of PS under the LDPE islands. Averaging the data obtained for the two modes, the indentation moduli of PANI and PANI-ND are retrieved and reported in Table 1.

We observe that the results obtained by TH-AFM and CR-AFM are in fairly good agreement, which represents a crossvalidation of the techniques. Surface indentation moduli retrieved with the two AFM based techniques have been compared to the bulk ones measured by standard nanoindentation. The latter are reported together with the measured indentation hardness $\left(H_{s}\right)$ in Table 1 , obtained with penetration depth varying between $500 \mathrm{~nm}$ and $1.2 \mu \mathrm{m}$, where the experimental uncertainties is related to the not homogeneity of the samples. Bulk indentation moduli are significantly higher for both the samples, although the relative variation of modulus due to the introduction of NDs is almost the same for all the three techniques. In particular, bulk indentation modulus of PANI is in good agreement with the value we have previously determined by AFM nanoindentation [5]. It is well known that mechanical properties of the surface of polymers are decreased by the presence of a softer layer (a few nanometers thick) with reduced density [17]. Thus, our results indicate that, differently from standard indentation, which is sensitive to bulk mechanical properties, both TH-AFM and CR-AFM can probe the actual surface elastic modulus. This represents a further confirmation of the importance of AFM based techniques to measure mechanical properties at the nanoscale especially in case of $2 \mathrm{D}$ materials such as thin films and layers.

\section{Conclusion}

In conclusion, we have described two AFM based techniques for the characterizations of the surface indentation modulus of materials at the nanoscale, namely, TH-AFM and CR-AFM. They operating principles, the range of measurable sample indentation moduli, the main advantages and limitations have been discussed. Then, such techniques have been demonstrated in a particular case study, i.e., the nanomechanical characterization of polyaniline and polyaniniline doped with nanodiamond particles tablets prepared by a pressing process. The agreement between surface indentation moduli obtained with the two techniques represented a cross-validation of the methods. When compared to bulk indentation moduli retrieved with standard nanoindentation, we observed an almost constant ratio between the data for PANI and PANI-ND. Moreover, surface indentation moduli measured with the AFM based methods are significantly lower than the bulk ones measured with nanoindentation, which can be attributed to the presence of nanometrical surface layers of polymer softer than the bulk. Overall, the results confirm the importance of methods such the AFM based ones for the mechanical testing at the nanoscale, which are capable of investigating ultrathin films and layers out of the range of standard mechanical tests. 


\section{References}

1. C. Reynaud, F. Sommer, C. Quet, N. El Buonia, D. Tran Minh, Surf. Interface Anal. 30, 185 (2000)

2. T. Kocourek, M. R`ǔzek, M. Landa, M. Jel'inek, J. Mik`sovsk`y, J. Kope`cek, Surf. Coat. Tech. 205 , S67 (2011)

3. Y.H. Ding, X.H. Deng, X. Jiang, P. Zhang, J.R. Yin, Y. Jiang, J. Mater. Sci. 48, 3479 (2013)

4. D. Passeri, A. Bettucci, A. Biagioni, M. Rossi, A. Alippi, E. Tamburri, M. Lucci, I. Davoli, S. Berezina, Ultramicroscopy 109, 1417 (2009)

5. D. Passeri, A. Alippi, A. Bettucci, M. Rossi, A. Alippi, E. Tamburri, M.L. Terranova, Synth. Met. 161, 7 (2011)

6. S.N. Magonov, D.H. Reneker, Annu. Rev. Mater. Sci. 27, 175 (1997)

7. S. Amelio, A.V. Goldade, U. Rabe, V. Scherer, B. Bhushan, W. Arnold, Thin Solid Films 392, 75 (2001)

8. M. Prasad, M. Kopycinska, U. Rabe, W. Arnold, Geophys. Res. Lett. 29, 1172 (2002)

9. D. Passeri, A. Bettucci, M. Germano, M. Rossi, A. Alippi, V. Sessa, A. Fiori, E. Tamburri, M.L. Terranova, Appl. Phys. Lett. 88, 121910 (2006)

10. M. Kopycinska-M“uller, R.H. Geiss, J. M“uller, D.C. Hurley, Nanotechnology 16, 703 (2005)

11. P.A. Yuya, D.C. Hurley, J.A. Turner, J. Appl. Phys. 104, 074916 (2008)

12. J.P. Killgore, D.G. Yablon, A.H. Tsou, A. Gannepalli, P.A. Yuya, J.A. Turner, R. Proksch, D.C. Hurley, Langmuir 27, 13983 (2011)

13. P.A. Yuya, D.C. Hurley, J.A. Turner, J. Appl. Phys. 109, 113528 (2011)

14. P. Sch“on, S. Dutta, M. Shirazi, J. Noordermeer, G.J. Vancso, J. Mater. Sci. 46, 3507 (2011)

15. P. Sch“on, K. Bagdi, K. Moln’ar, P. Markus, B. Puk’anszky, G.J. Vancso, Eur. Polym. J. 47, 692 (2011)

16. M.E. Dokukin, I. Sokolov, Langmuir 28, 16060 (2012)

17. D. Passeri, M. Rossi, E. Tamburri, M.L. Terranova, Anal. Bioanal. Chem. 405, 1463 (2013)

18. E. Tamburri, V. Guglielmotti, S. Orlanducci, M.L. Terranova, D. Sordi, D. Passeri, R. Matassa, M. Rossi, Polymer 53, 4045 (2012)

19. E. Tamburri, V. Guglielmotti, S. Orlanducci, M.L. Terranova, D. Passeri, M. Rossi, D. Sordi, in MRS Proceedings, vol. 1452 (Cambridge Univ Press, 2012), vol. 1452, pp. mrss12-1452-ff05-06

20. M.L. Terranova, E. Tamburri, V. Guglielmotti, F. Toschi, S. Orlanducci, D. Passeri, M. Rossi, AIP Conf. Proc. 1459(1), 111 (2012)

21. D. Passeri, A. Biagioni, M. Rossi, E. Tamburri, M.L. Terranova, Eur. Polym. J. 49, 991 (2013)

22. E. Tamburri, V. Guglielmotti, R. Matassa, S. Orlanducci, S. Gay, G. Reina, M.L. Terranova, D. Passeri, M. Rossi, J. Mater. Chem. C 2(19), 3703 (2014)

23. O. Sahin, S. Magonov, C. Su, C.F. Quate, O. Solgaard, Nat. Nanotechnol. 2, 507 (2007)

24. O. Sahin, N. Erina, Nanotechnology 19, 445717 (2008)

25. B.V. Derjaguin, V.M. Muller, Y.P. Toporov, J. Colloid Interface Sci. 53, 314 (1975)

26. P. Ihalainen, J. J“arnstr“om, A. M“a”att”anen, J. Peltonen, Colloid. Surface. A 373, 138 (2011)

27. K. Sweers, K. van der Werf, M. Bennink, V. Subramaniam, Nanoscale Research Letters 6, 270 (2011)

28. K.M. Leung, G. Wanger, Q. Guo, Y. Gorby, G. Southam, W.M. Laue, J. Yang, Soft Matter 7, 6617 (2011)

29. U. Rabe, J. Janser, W. Arnold, Rev. Sci. Instrum. 67, 3281 (1996)

30. D. Passeri, A. Bettucci, M. Germano, M. Rossi, A. Alippi, S. Orlanducci, M.L. Terranova, M. Ciavarella, Rev. Sci. Instrum. 76, 093904 (2005)

31. S. Jena, R.B. Tokas, N. Kamble, S. Thakur, D. Bhattacharyya, N.K. Sahoo, Thin Solid Films 537, 163 (2013)

32. G. Stan, W. Price, Rev. Sci. Instrum. 77, 103707 (2006)

33. D. Passeri, M. Rossi, J.J. Vlassak, Ultramicroscopy 128, 32 (2013)

34. U. Rabe, in Applied scanning probe methods II, ed. by B. Bhushan, H. Fuchs, pp. 37-90

35. U. Rabe, M. Kopycinska-M“uller, S. Hirsekorn, in Acoustic scanning probe microscopy, ed. by F. Marinello, D. Passeri, E. Savio (Springer

Verlag (Berlin, Heidelberg), 2012), chap. 5, pp. 123-153

36. D.C. Hurley, J.A. Turner, J. Appl. Phys. 102, 033509 (2007)

37. M. Kopycinska, C. Ziebert, H. Schmitt, U. Rabe, S. Hirsekorn, W. Arnold, Surf. Sci. 532, 450 (2003)

38. Y. Zheng, R.E. Geer, K. Dovidenko, M. Kopycinska-M“uller, D.C. Hurley, J. Appl. Phys. 100, 124308 (2006)

39. G. Stan, C.V. Ciobanu, T.P. Thayer, G.T. Wang, J.R. Creighton, K.P. Purushotham, L.A. Bendersky, R.F. Cook, Nanotechnology 20, 035706 (2009)

40. M. Preghenella, A. Pegoretti, C. Migliaresi, Polym. Test. 25, 443 (2006)

41. D. Passeri, M. Rossi, A. Alippi, A. Bettucci, M.L. Terranova, E. Tamburri, F. Toschi, Physica E 40, 2419 (2008)

42. W. Zhao, R.P. Singh, C.S. Korach, Compos. Part A - Appl. S. 40, 675 (2009)

43. D.G. Yablon, A. Gannepalli, R. Proksch, J. Killgore, D.C. Hurley, J. Grabowski, A.H. Tsou, Macromolecules 45, 4363 (2012)

44. A. Fava, M. Lucci, D. Faso, A. Luzzi, M. Salvato, A. Vecchione, R. Fittipaldi, I. Ottaviani, I. Colantoni, M. Tomellini, I. Davoli, J. Electrochem. Soc. 161, D540 (2014)

45. S.K. Balijepalli, I. Colantoni, R. Donnini, S. Kaciulis, M. Lucci, R. Montanari, N. Ucciardello, A. Varone, La Metallurgia Italiana 1/2013, 42 (2013)

46. J.P. Killgore, J.Y. Kelly, C.M. Stafford, M.J. Fasolka, D.C. Hurley, Nanotechnology 22(17), 175706 (2011) 
Table 1 Indentation modulus values of PANI and PANI-ND obtained with TH-AFM, CRAFM, and standard nanoindentation. For the sake of completeness, values of sample indentation hardness $H_{s}$ retrieved through nanoindentation are also reported.

\begin{tabular}{ccccc}
\hline & TH-AFM & CR-AFM & \multicolumn{2}{c}{ Nanoindentation } \\
Samples & $\mathrm{M}_{\mathrm{S}}[\mathrm{GPa}]$ & $\mathrm{M}_{\mathrm{S}}[\mathrm{GPa}]$ & $\mathrm{M}_{\mathrm{S}}[\mathrm{GPa}]$ & $130 \pm 60$ \\
\hline PANI & $1.4 \pm 0.2$ & $1.2 \pm 0.1$ & $4.3 \pm 1.4$ & $12.0 \pm 5.0$ \\
PANI-ND & $2.3 \pm 0.5$ & $3.1 \pm 0.5$ & $1200 \pm 200$ & $300 \pm$ \\
\hline
\end{tabular}

Table 2 Calculated and measured values of the first four flexural resonance frequencies of the cantilever used in CR-AFM.

\begin{tabular}{lll}
\hline Frequency & Calculated $[\mathrm{kHz}]$ & Measured [kHz] \\
$\mathrm{f}_{01}$ & 19 & 26.7 \\
$\mathrm{f}_{02}$ & 120 & 169.8 \\
$\mathrm{f}_{03}$ & 341 & 477.3 \\
$\mathrm{f}_{04}$ & 664 & 933.0 \\
\hline
\end{tabular}

Fig. 1 Sketch of two models used to analyze CR-AFM data: (a) normal spring coupled cantilever, where lateral forces, tip height and cantilever tilt angle are neglected; (b) normal and tangential spring coupled cantilever.

Fig. 2 Topographical images of PANI ( $a$ and $b$ ) and PANI-ND (c and d). Polynomial background has been subtracted to highlight the nanoscale features of the surfaces.

Fig. 3 Histograms of the indentation moduli measured on the PS-LDPE (red), PANI-ND (green) and PANI (blue) samples (symbols) together with the corresponding Gaussian fit (solid lines).

Fig. 4 (a) CR-AFM map of the first contact resonance frequency acquired on the PS-LDPE reference sample and (b) profile extracted in correspondence of the dashed line.

Fig. 5 First (blue lines) and third (red lines) contact resonance frequencies as a function of the normalized contact stiffness $k^{*} / k_{c}$. The dotted lines were calculated considering the simple clamped spring-coupled cantilever, i.e, the model in Fig. 1a. The solid curves are obtained introducing the contribution of a lateral spring $\left(k_{\text {lat }}^{*}\right)$, i.e, the model in Fig. $1 \mathrm{~b}$. 
Figure 1

a

SAMPLE SURFACE

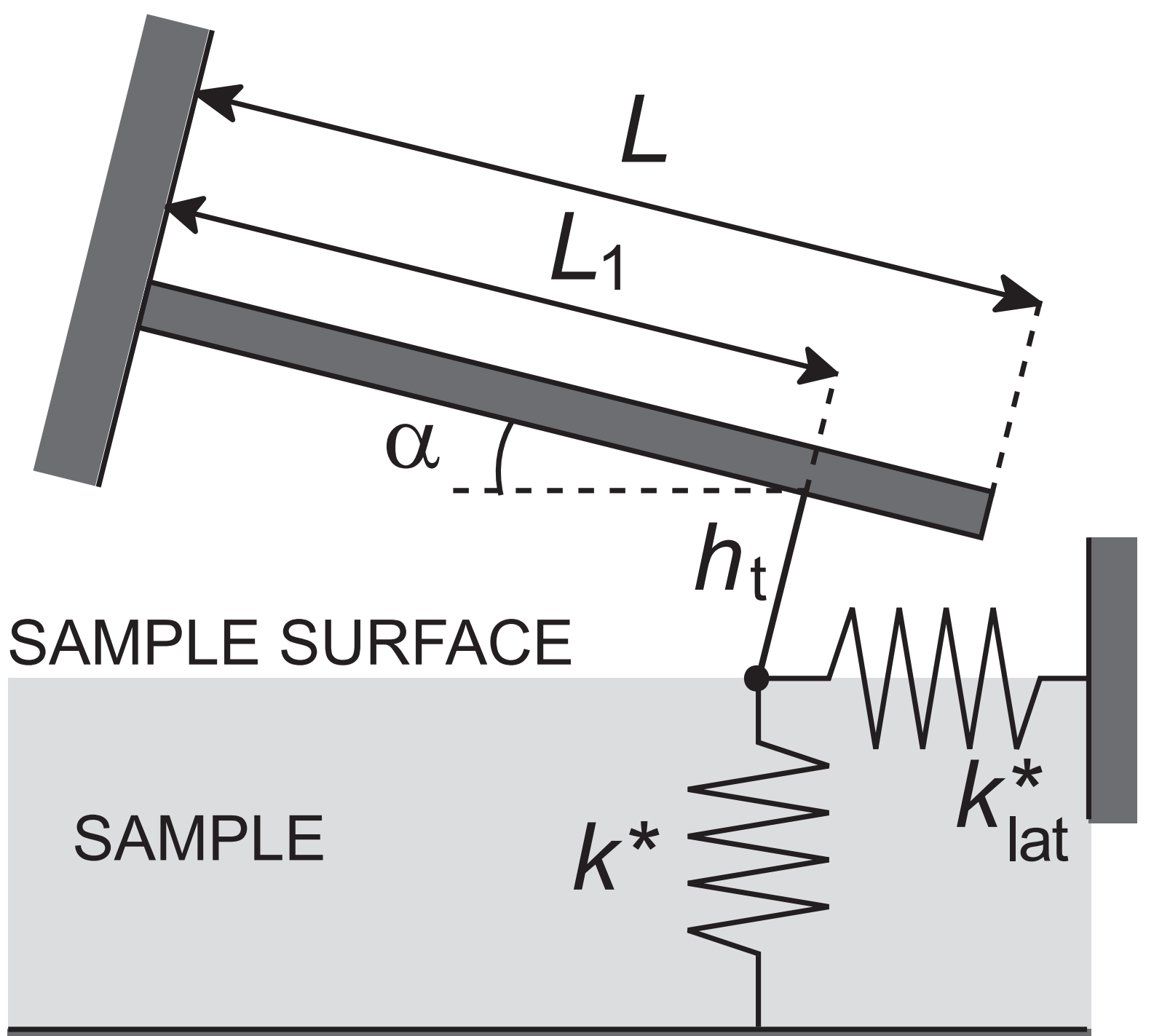


a
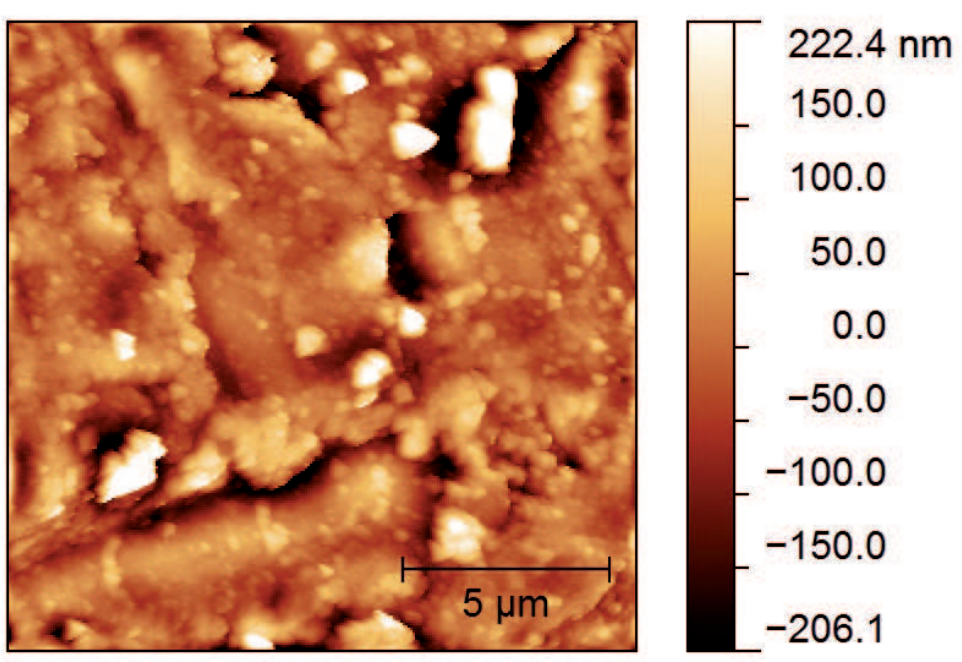

C
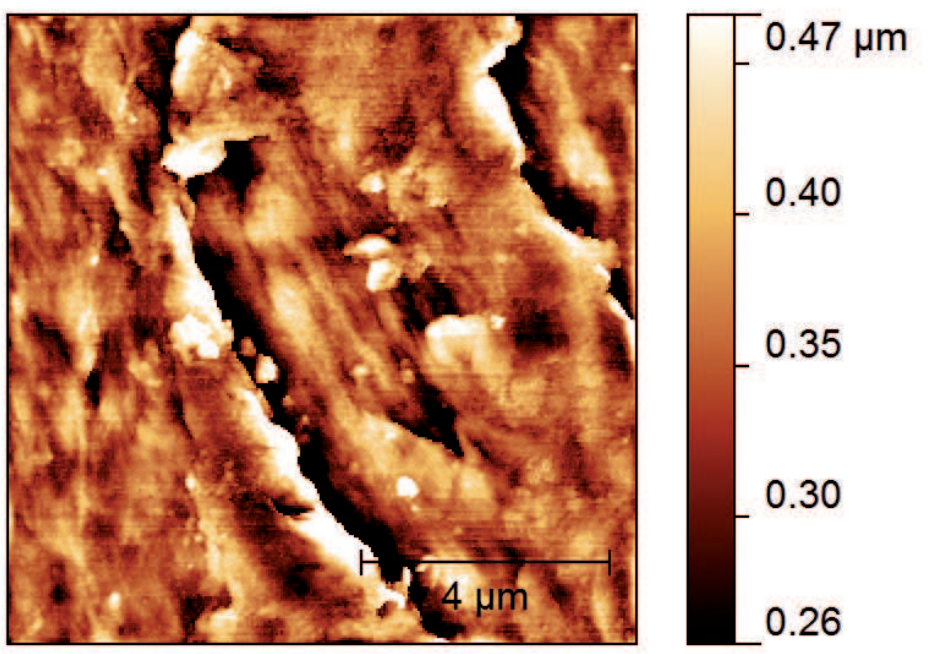

b

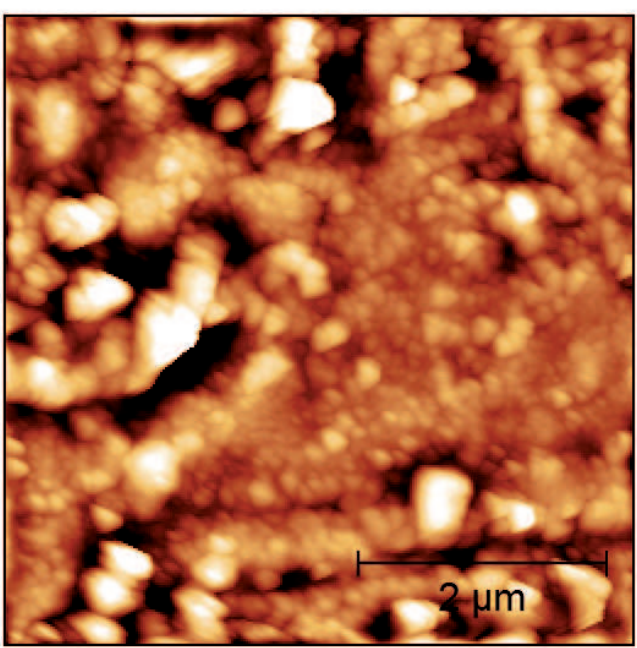

\begin{tabular}{|l}
$155.5 \mathrm{~nm}$ \\
140.0 \\
130.0 \\
120.0 \\
110.0 \\
100.0 \\
90.0 \\
80.0 \\
70.0 \\
61.2
\end{tabular}

d
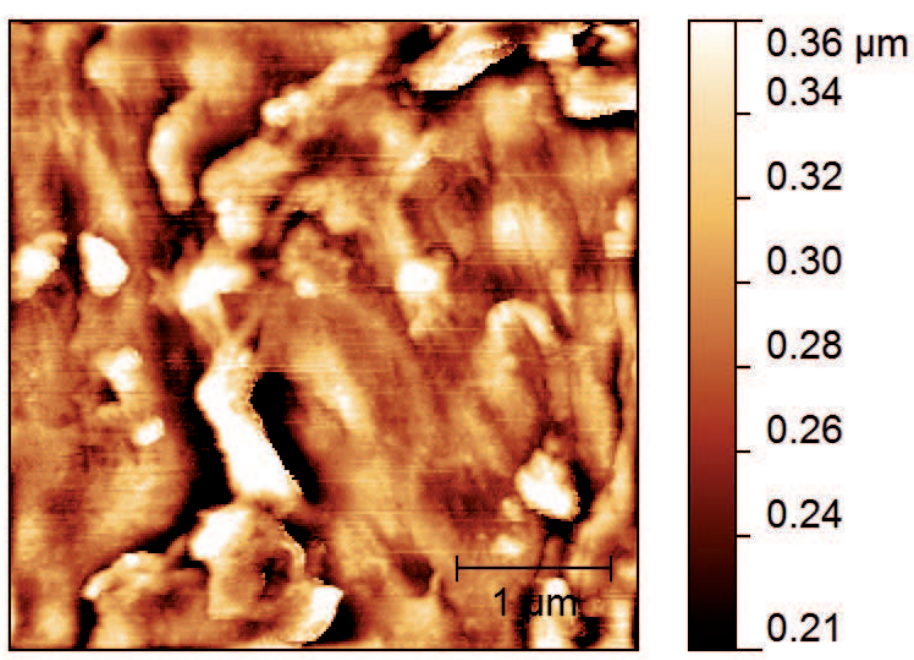
Figure 3

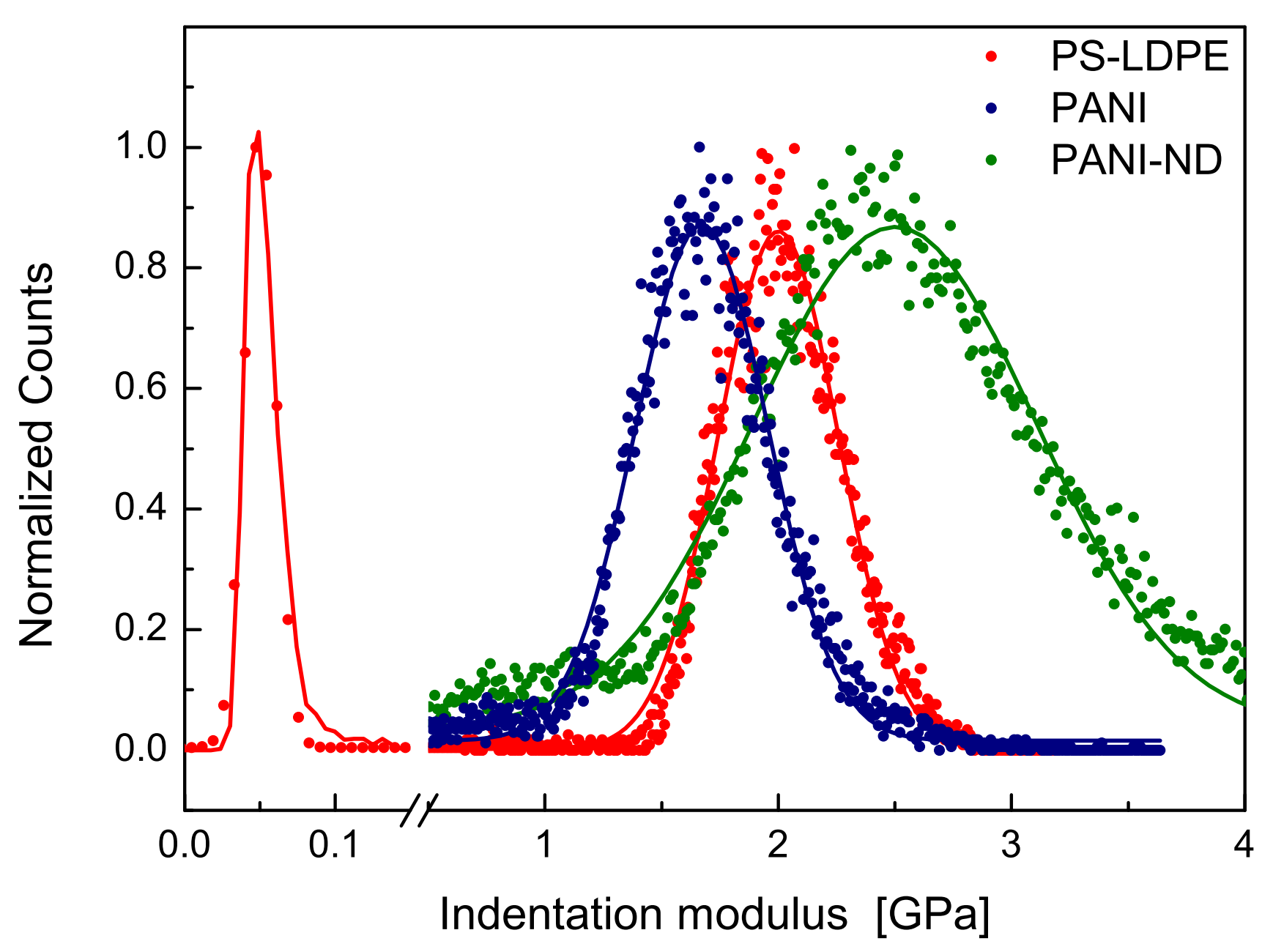



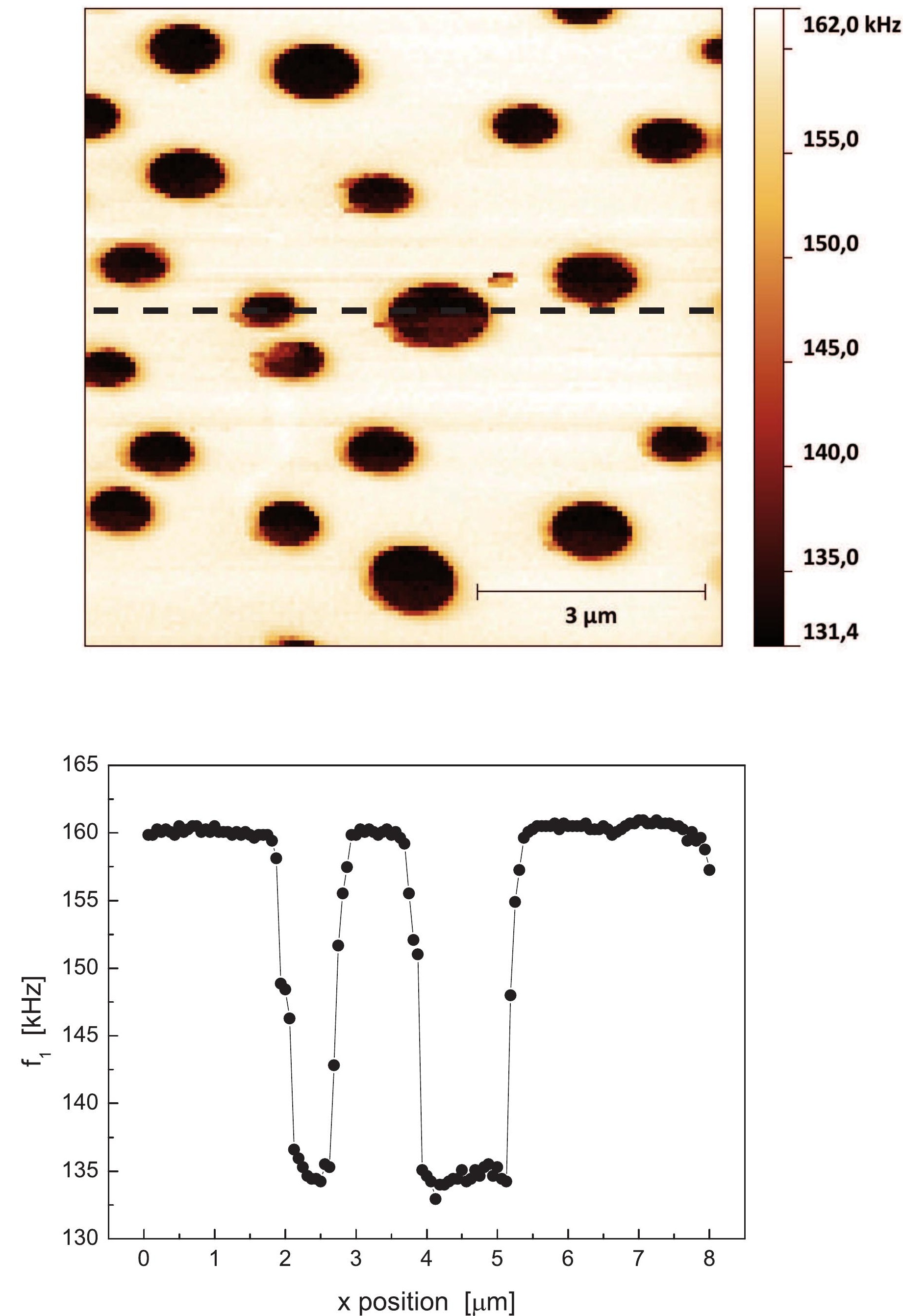

b 
Figure 5

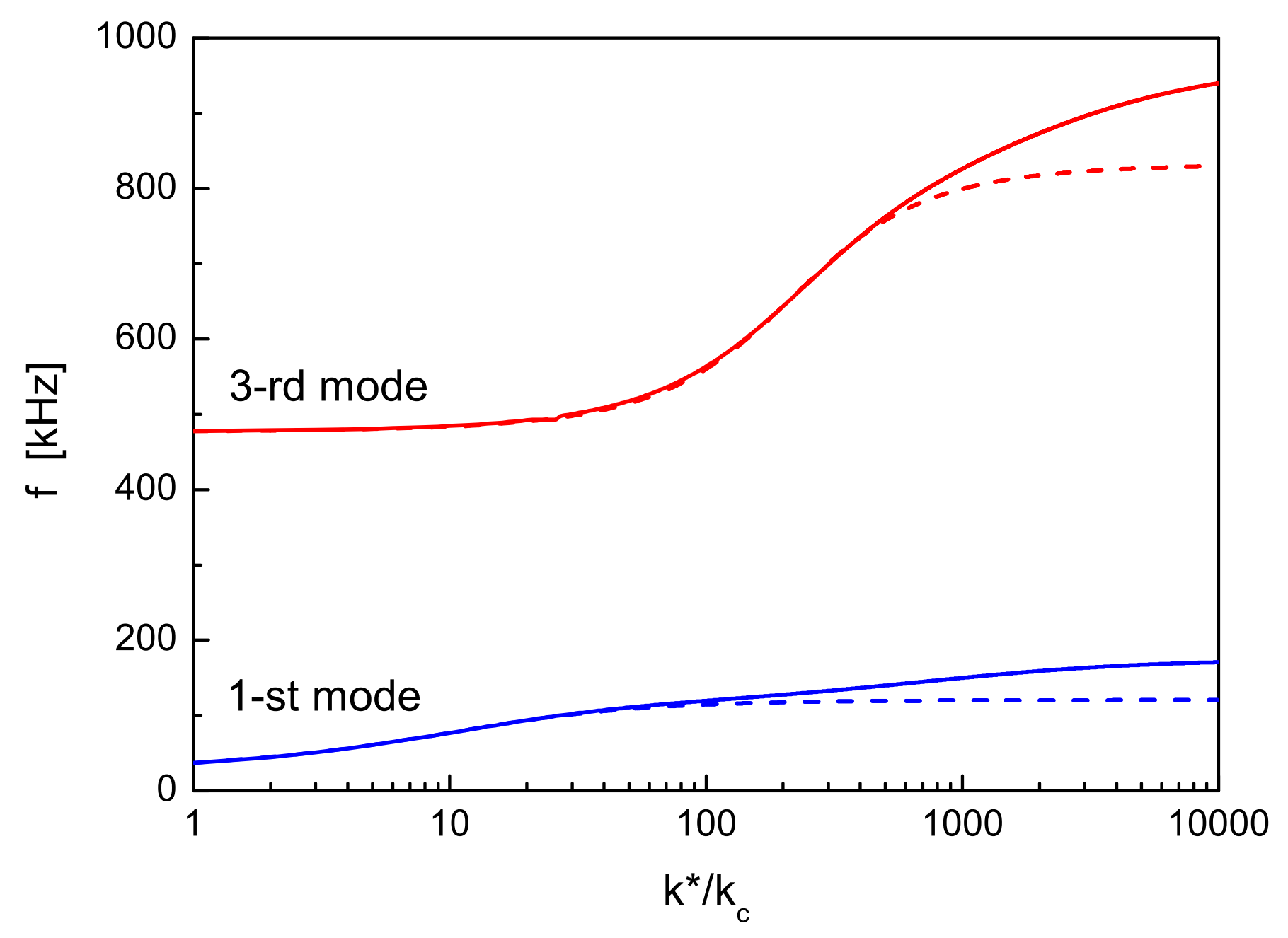

